\title{
Automatic Adjustment of Manually Measured QT Intervals in Digital Electrocardiograms Improves Precision of Electrocardiographic Drug Studies
}

\author{
K Hnatkova, M Malik \\ St. George's Hospital Medical School, London, United Kingdom
}

\begin{abstract}
Drug investigating studies require similar patterns of $T / U$ waves to be measured at the same fiducial points. However, such a systematic measurement is difficult to achieve with manual measurement as compulsory for regulatory review.

A new system was developed that adjusts manually measured QT intervals in sets of ECGs obtained from the same drug study. For a given threshold $w$, the system organizes all the repolarisation patterns in groups of ECG leads that correlate each with each other with abs $(r)>w$. Within these groups, the system assigns a shift of the measured $T$ wave end in each ECG lead to synchronise the patterns of the manual measurements. The same approach is used to align the QRS onset measurements.

The system was used in a drug-study of 4090 ECGs obtained in healthy volunteers off-and on-treatment with an investigational drug. The difference between manual measurements and the measurements adjusted by the new software was characterised by calculating the regression residua between $Q T$ and $R R$ intervals and by comparing the QT dispersion. After applying the system, the regression residua were reduced from 15.06 to $14.32 \mathrm{~ms}$ $(p<0.002)$. The $Q T$ dispersion values were reduced from $38.3(S D=17.2)$ to $30.2(S D=15.4) \mathrm{ms}\left(p<10^{-200}\right)$.

Automatic adjustment of manual measurements of $Q T$ intervals in electronically recorded ECGs improves the precision of $Q T$ interval assessment in electrocardiographic drug studies.
\end{abstract}

\section{Introduction}

The manual detection of the $\mathrm{T}$ wave offset is known to be frequently rather unreliable [1,2] but the automatic measurement of QT interval [3] still remains a challenging problem. There were many studies comparing the performance of manual and automatic measurement of QT interval [4,5]. The automatic QT measurement have been reported to be more stable and better reproducible than manual measurement [6] especially when dealing with electrocardiograms (ECG) that do not show gross repolarisation abnormalities.

A precise automatic detection of the end of $\mathrm{T}$ or $\mathrm{T} / \mathrm{U}$ wave pattern has been the subject of many investigations that involved different approaches ranging from the simple algorithms such as the intercept of the slope and baseline [7] to more complicated concepts such as the algebraic decomposition of $\mathrm{T}$ and $\mathrm{T} / \mathrm{U}$ wave patterns into individual action potential-like elementary constituents [8]. Some believe that the assessment of the duration of the ventricular repolarisation is not the correct way forward when handling electronic signals and investigate entirely new descriptors of electrocardiographic repolarisation patterns $[9,10]$.

Both manual and automatic measurements still suffer from errors caused by a low $\mathrm{T}$ wave amplitude, or by merges of $\mathrm{T}$ wave with $\mathrm{U}$ and/or $\mathrm{P}$ wave. The major practical problem with automatic measurements is that it occasionally leads to very gross errors, e.g. when the algorithm is influenced by noise or by unusually shaped repolarisation abnormalities. This is especially important for the studies addressing drug-induced repolarisation changes that are used as surrogates of proarrhythmic toxicity. Such studies frequently involve data processing of thousands ECGs, and require very reliable measurements. Because of the danger of false negative reporting of unusual QT interval prolongations, regulatory agencies require the use of fully manual measurements for the assessment of drugs with potential repolarisation involvement [11-13].

At the same time, regulatory distinctions between drug safety and potential proarrhythmic toxicity are frequently based on studies reporting only minute QT interval prolongations that are comparable to, if not smaller than the precision achievable with the manual measurement of QT intervals. A systematic approach with the measurement of fairly similar $\mathrm{T}$ and $\mathrm{T} / \mathrm{U}$ wave patterns to the very same position of the fiducial point is needed. However, such a strictly systematic methodology is practically impossible to achieve with purely manual 
positioning of measurement cursors.

Therefore a system synchronising the positions of manually located measurement cursors in similar $\mathrm{T}$ and $\mathrm{T} / \mathrm{U}$ wave patterns has been developed.

\section{Method}

A new system has been developed that adjusts manually measured QT intervals in sets of 12 leads ECG recordings.

Let us denote $\varepsilon^{\lambda}\left(t_{j}\right), \lambda=1, \ldots, 12, j=0, \ldots, e, t_{j}=t_{j-1}+\Delta t$, $0<j \leq e$, the digital value of $\lambda$ lead of ECG $\varepsilon$ in equally distributed discrete time instances $t_{j}$. Symbols $t_{0}$, and $t_{e}$ denote time instants of the beginning, and the end of the ECG recording, respectively. This study used the following constant values $t_{0}=0, t_{e}=1200 \mathrm{~ms}$, and $\Delta t=2 \mathrm{~ms}$ in all ECG recordings. A set of all leads of all ECGs $\Sigma=\left\{\varepsilon_{i}^{\lambda}\right\}_{i=1, \lambda=1}^{i=n, \lambda=12}$ was decomposed into $k$ different subsets $\sigma$ of individual ECG leads such that $\Sigma=\bigcup_{j=0}^{k} \sigma_{j}$, where $k$ is the number of all subsets, and the condition $\bigcap\left(\sigma_{j}, \sigma_{j j}\right)=\varnothing$ is fulfilled for each $j, j j=0, \ldots, \mathrm{k}, j \neq j j$.

The symbols $Q\left(\varepsilon^{\lambda}\right)$, and $T\left(\varepsilon^{\lambda}\right)$ will be used further for the time instants of manually measured values of QRS onset, and T wave offset, respectively in lead $\lambda$ of ECG $\varepsilon$. They are mapping set $\Sigma$ on a set $R$ of real positive numbers. For each function $f=Q, T$, there is a separate subset $\bar{\Sigma}^{f}, \bar{\Sigma}^{f} \subseteq \Sigma$ containing all leads that were not manually measurable (either the lead was too noisy, or the morphology was so unclear that the manual detection of corresponding value was impossible, etc.). Let us always further denote $\sigma_{0}=\bar{\Sigma}^{f}, \quad \bar{\Sigma}^{f} \subseteq \Sigma$. The decomposition algorithm selected the first available lead $\alpha \in \tilde{\Sigma}, \tilde{\Sigma}=\Sigma \backslash \sigma_{0}$ to create a subset $\sigma_{1}^{\alpha}$. The symbol $\tilde{\Sigma}$ will be further used for the set of all unprocessed leads. Subsequently, all leads $\beta \in \Sigma \backslash\left(\bigcup\left(\sigma_{0}, \sigma_{1}^{\alpha}\right)\right.$ were correlated with lead $\alpha$ within a window starting $w_{s} \mathrm{~ms}$ prior to, and ending $w_{e}$ ms after the manually measured point $p$ (where $p$ stands for either QRS onset or T wave offset). This window was shifted $\pm h$ ms (with 2 ms step) around $p$. The value of $w_{s}$ was set-up to $60 \mathrm{~ms}$ for Q onset and $200 \mathrm{~ms}$ for $\mathrm{T}$ wave offset. The value of $w_{e}$ was set-up to $80 \mathrm{~ms}$ for Q onset and $100 \mathrm{~ms}$ for T offset. The shift $h$ was used 40, and $80 \mathrm{~ms}$ around QRS onset and $\mathrm{T}$ wave offset, respectively. The Pearson correlation coefficient $\wp(x, X, y, Y)$ was used to correlate lead $X$ and lead $Y$ within windows around the time instant $x$, and $y$, respectively. The shift $\mu(\beta)=t$, corresponding to the maximum correlation $\Re(0, \alpha, t, \beta)=\max _{t=-h, t=t+\Delta t}^{t=+h}(|\wp(0, \alpha, t, \beta)|) \quad$ was evaluated for each pair of leads $\alpha$, and $\beta$. All leads $\beta$ for which $\mathfrak{R}(0, \alpha, \mathrm{t}, \beta) \geq \tau, \quad \tau$ was selected threshold, were included in a subset $\sigma_{1}^{\alpha}$. Subsequently, the correlation was checked for each lead $\lambda_{1} \in \sigma_{1}^{\alpha}, \lambda_{1} \neq \alpha$ and each lead $\lambda_{2} \in \sigma_{1}^{\alpha}, \quad \lambda_{2} \neq \alpha, \quad \lambda_{1} \neq \lambda_{2}$, within the window aligned according to the shifts $\mu\left(\lambda_{1}\right), \mu \quad\left(\lambda_{2}\right)$. If $\left|\wp\left(\mu\left(\lambda_{1}\right), \lambda_{1}, \mu\left(\lambda_{2}\right), \lambda_{2}\right)\right|<\tau$, lead $\lambda_{2}$ was returned to the set $\tilde{\Sigma}$ of all unprocessed leads. The algorithm was repeated until $\tilde{\Sigma}=\varnothing$. Obviously, each step of the decomposition created a subset $\sigma_{i}^{\lambda}$, containing at least one lead $\lambda$. Therefore, the algorithm stopped after final number of steps and performed decomposition described above.

The number of decomposed groups $\sigma_{i}$ is dependent on the initial order of the ECG patterns. Therefore, the stability of the algorithm and its dependency on the initial orders was investigated. The analysis was repeated for several $(n=100)$ orders of the original data and the shifts assigned to measured $\mathrm{T}$ wave ends are averaged in each ECG lead.

Following the decomposition, all leads in each subset $\sigma_{i}$ were aligned according to the average shift of subset $\sigma_{i}^{\lambda}$, and synchronised values of QRS onset and T offset points were evaluated. In that way, each subset $\sigma_{i}$ contained similar patterns of either QRS complex or T wave morphology.

The difference between the initial manual measurements and the measurements adjusted by the system was characterised by calculating the regression residua between QT and RR intervals. The logarithmic $\mathrm{QT} / \mathrm{RR}$ model $\mathrm{QT}=b+a \times \ln (\mathrm{RR})$, where $a$, and $b$ are parameters of the model, was used in this comparison. The regression residuum has been calculated as a standard deviation of the heart rate corrected QT interval $\mathrm{QT} c=\mathrm{QT}-a \times \ln (\mathrm{RR})$. The corresponding QT dispersion (range of measured QT interval within the same ECG) was calculated and this comparison was taken as an expression of measurement imprecision.

\section{ECG recordings}

The system has been used in a drug study involving a set of $n=409012$ leads ECGs of $10 \mathrm{sec}$ recordings obtained from healthy female volunteers off- and ontreatment with an investigation drug. Only ECGs with at least 6 measurable leads and with stable RR interval sequence within the recording were included in this study.

\section{Results}

The manual measurements in total number of 3551 out of 4090 ECGs were adjusted using this system. The 
remaining 539 ECGs were excluded from the study: 424 were measurable in less than 6 leads, and 115 revealed unstable duration of RR interval within ECG recording.

The figure 1 shows the scatter plots of the QT/RR relationship of the original manual measurement and measurement adjusted by the system. While the system increased QT values for lower RR values, higher RR values resulted in decrease of QT values. In general, the QT/RR cloud became "more compact" after application of the system. The slope of the logarithmic QT/RR model (parameter a) reduced from 0.1327 for manual measurement to 0.1276 for adjusted measurement.

The improved precision of the system was reflected by the regression residua that were reduced from 15.06 to $14.32 \mathrm{~ms} \quad(\mathrm{p}<0.002$ - paired two-sided t-test). Similar trend was observed for the QT dispersion values that were reduced from 38.4 (standard deviation 17.2) to 30.2 (standard deviation 15.4$) \mathrm{ms}\left(\mathrm{p}<3.5 \times 10^{-284}\right.$ - paired twosided t-test).

The algorithmic stability was tested in the total number of $n=100$ iterations of the original order of leads in the set $\tilde{\Sigma}$. The adjustment shifts derived from the first and last 50 iterations of the system were highly correlated $(\mathrm{r}=0.867)$ confirming the algorithmic stability of the system.

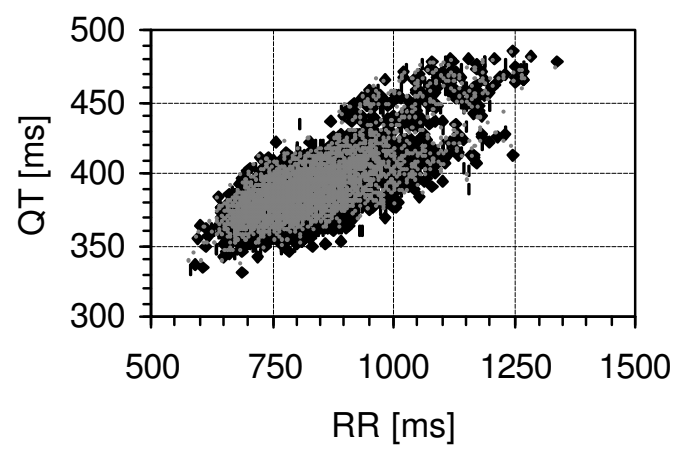

Figure 1.

Figure shows the scatter plots of QT/RR relationship. Each ECG recording is represented as a pair of mean RR interval and median value of QT intervals measured at least 6 leads of ECG. Manual and adjusted measurement of QT values is shown in black diamonds and gray dots, respectively.

\section{Conclusion}

The prolongation of the electrocardiographic QT interval is one of the tests that can stop the development of a new drug. Clinical assessment of drug-induced QT interval prolongation is crucially dependent on the quality of electrocardiographic data and the appropriateness of electrocardiographic analyses [12].

This study demonstrates the possibility of automatic adjustment of manual measurement QT intervals in order to receive maximally synchronise measurements for similar $\mathrm{T}$ or $\mathrm{T} / \mathrm{U}$ waves patterns. The small and high $\mathrm{T}$ wave amplitudes are subjected to the same approach directed by the simple calculation of correlation coefficient. Therefore, the improved and synchronised precision of QT measurement can be achieved in electronically recorded ECGs for the purposes of drug related studies.

\section{References}

[1] Taran L, Szilagyi N. The measurement of the Q-T interval in acute heart disease. Bull St. Francis Sanatorium 1951;813.

[2] Murray A, McLaughlin NB, Bourke JP, Doig JC, Furniss SS, Campbell RWF. Errors in manual measurement of QT intervals. Br Heart J 1994;71:386-90.

[3] Malik M, Batchvarov VN. Measurement, Interpretation and Clinical Potential of QT dispersion. J Am Coll Cardiol. 2000; 36: 1749-66.

[4] McLaughlin NB, Campbell RWF, Murray A. Accuracy of four automatic QT measurement techniques in cardiac patients and healthy subjects. Heart 1996;76:422-426.

[5] Murray A, McLaughlin NB, Campbell RWF. Measuring QT dispersion: man versus machine. Heart 1997;77:539542.

[6] Savelieva I, Yi G, Guo X, Hnatkova K, Malik M. Agreement and reproducibility of automatic versus manual measurement of QT interval and QT dispersion. Am J Cardiol 1998;81:471-477.

[7] Lepeschkin E, Surawicz B. The measurement of the Q-T interval in electrocardiogram. Circulation 1952;VI:378388.

[8] Padrini R, Butrous G, Statters D, Camm AJ, Malik M. Morphological algebraic models of the TU-wave patterns/in idiopathic long QT syndrome. Int J Cardiol 2001;77:151-62.

[9] Acar B, Yi G, Hnatkova K, Malik M. Spatial, temporal and wavefront direction characteristics of 12-lead T-wave morphology. Med Biol Eng Comput 1999;37(5):574-84.

[10] Kors JA, de Bruyne MC, Hoes AW, van Herpen G, Hofman A, van Bemmel JH, Grobbee DE. T axis as an indicator of risk of cardiac events in elderly people. Lancet 1998;352(9128):601-5.

[11] Sheridan DJ. Drug-induced proarrhythmic effect: assessment of changes in QT interval. Br J Clin Pharmacol 2000;50:297-302.

[12] Gralinski MR. The assessment of potential for QT interval prolongation with new pharmaceuticals: impact on drug development. J Pharmacol Toxicol Methods 2000;43(2):91-9.

[13] Malik M, Camm AJ. Evaluation of drug-induced QT interval prolongation: implications for drug approval and labelling. Drug Saf 2001;24(5):323-51. 
Address for correspondence.

Katerina Hnatkova,

St. George's Hospital Medical School,

Department of Cardiological Sciences,

Cranmer Terrace,
London,

SW17 ORE,

United Kingdom

E-mail address: k.hnatkova@sghms.ac.uk 